\title{
Remission rates of select mental illness and substance use disorders in India
}

\section{Anusa A.M*}

Department of Psychiatry, Shri

Satya Sai Medical College and

Research Institute, Sri Balaji

Vidyapeeth, Ammapettai,

Kanchipuram District,

Tamilnadu - 603108.

For Correspondence

${ }^{*}$ Dr. Anusa A.M,

Email: anusamd@gmail.com

Date of

Submisssion: 12-01-2019

Acceptance: 26-02-2019

Access this article online

Quick Response Code

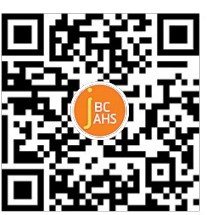

https://www.jbcahs.org

E-ISSN: 2581-6039

\section{ABSTRACT}

Background and Objective: There are very few literature evidence studying the remission rates among mental illness and substance use disorders (MISUD) in India. Knowledge of the remission and its characteristics can help to draw better policies for mental health care delivery in India.

Material and Methods: Using the Global Burden of Diseases, 2016 approach and employing the DisMod-MR - 2.1.6 mathematical modelling, prevalence, incidence and remission rates of 4 substance use disorders (alcohol, opioid, cocaine and cannabis), schizophrenia, major depressive disorder, bipolar disorder and anxiety disorders were studied.

Results: Within the mathematical model confines the prevalence, incidence characteristics of MISUDs based on age and gender are presented. The remission rate per person year ranged from 0.015 to 1.46 for different MISUD. Gender shows difference in the model for anxiety disorders, opioid and alcohol use disorder. The effect of age on the model are described in detail

Conclusion: The outcome of the study indicates the remission rates of MISUDs are highly variable. Remission rates significantly varies between age and gender. Based on these inputs, customized and targeted MISUDs therapies may be designed for better remission. The study also underlines the need for further large scale research efforts required for studying and reporting natural history of MISUDs from India.

\section{Keywords:}

Remission rate, mental illness, substance use disorder, epidemiology, prevalence

\section{INTRODUCTION}

Mental illness and substance use disorder (MISUD) includes a spectrum of heterogeneous, multifactorial diseases with diverse presentations, occurrence patterns and treatments. ${ }^{1}$ Resultantly, the outcome of MISUDs are often complex. The outcomes are often described as episodic, response, partial remission, full remission, recovery, relapse, and recurrence. ${ }^{2}$ The definitions for each of these are delineated separately for each disorder. Remission is often described to be achieved when the subject does not meet the criteria for the disorder and as in partial remission when the criteria is not met though in presence of minimal symptoms. ${ }^{2}$ Epidemiological estimation of remission is reportedly preferred for diseases that are chronic and variably progressive in nature, with MISUDs confirming to this standard, broad outline. ${ }^{3}$ Most of the studies in MISUD follow the natural history and or effect of various treatment, describe the failures of treatment or that emphasize on the progression of the disorders. Studies often include genetic predisposition, demographic, environmental, social and psychological factors that causes progression of the disorders. ${ }^{2}$ Interestingly remission is the least studied concept in MISUD. However, understanding remission rates for these conditions are crucial. Remission data of chronic episodic diseases could provide deeper insights on the standard of care, provide clue to point of care, primary care services and etiologic research too. ${ }^{2}$ J Basic Clin Appl Health Sci. 2019;2:14-24. 
Global Burden of Diseases (GBD) is a unique attempt to systematically analyze the huge volume of published peer-reviewed data, including mental health. With the proven power of mathematical modelling and consideration of various parameters using the power of rapid computation, this project is widely used to combine and provide more meaningful insights of data from several small scale studies. ${ }^{4-15}$

The recently concluded National Mental Health Survey of India revealed a deep insights of the prevalence, incidence, burden of mental illness, availability of resources and treatment gap in several parts of India. ${ }^{1}$ Together with ever increasing body of MISUDs literature from India, more knowledge is continuously and incrementally emerging in the field of MISUD. However, less pertinent data is available in the dimensions of remission. ${ }^{16}$ This manuscript intends to address this lacunae. This observational study intends to estimate the prevalence, incidence of select common MISUDs in India in 2016 and calculate the remission rates for the conditions using the GBD approach as well as to study the effect of age and gender on the remission.

\section{MATERIAL AND METHODS}

From the Indian Mental Health Survey, we identified that the most common MISUD in India were schizophrenia, bipolar disorders, major depressive disorder, anxiety and substance (alcohol dependence, cannabis, cocaine and opioid) use disorders. ${ }^{1}$ The present study reports of the projected estimates for these disorders only for the year 2016.

The data used in the study was obtained from the the GBD study (http://www.healthdata.org/datavisualization/gbd-compare). The data pertaining to the average duration of the disease was calculated from the data obtained by the author.

The GBD employs a standardized, complex mathematical, inclusive approach for various basic epidemiological and demographical parameters. They rely on published data, surveys and reliable public databases. The standard minimum inclusion criteria set of definition for MISUDs have been described previously elsewhere and the same set of definition were used for this study. ${ }^{15}$ All relevant India related publications from the past till 2016 have been used to collect the data, data extracted, optimized and enriched using established mathematical models described in details elsewhere. ${ }^{15}$
All collected data were combined using the Bayesian meta-regression tool DisMod-MR 2.1.16. This system encompass a series of alternative approach, as described in details earlier. ${ }^{15}$ The present study was performed for India at national level and estimates for 2016 are presented. Detailed results from GBD 2016 by type of MISUDs can be explored for basic combined burden parameters in dynamic data visualizations at https:// vizhub.healthdata.org/gbd-compare/

In situations, where there is a lack of sufficient quality data, to draw meaningful conclusions, the GBD uses a non-linear mixed effect model. In this model, the relationship between key covariates of the diseases and its associated rates, spatio-temporal regression, and Gaussian process regression are utilized to draw conclusions as well as to make prediction. GBD makes such predictions in cases where there are no or little observed data by borrowing strength from past data or with that of the pertinent neighboring countries that have empirical data. ${ }^{4-14}$ All previous published pertinent data from several sources as described in detail at http://ghdx.healthdata.org/about-ghdx/datatype-definitions.

A number of data points from several past years were identified and the collected data were used in this study from several sources studies. The combined data can be visualized at https://vizhub.healthdata.org/gbdcompare/. All computations in GBD were done 1000 times, every time drawing from the distribution of the sampling error of data inputs, the uncertainty of data corrections for measurement errors, the uncertainty in coefficients from model fit (DisMod-MR 2.1), and uncertainty bounds for a quantity of interest were defined by the $25^{\text {th }}$ and $975^{\text {th }}$ value of the ordered 1000 estimate values, expressed as the $95 \%$ uncertainty interval (UI)..$^{4-14}$

Using the GBD tool, the select MISUDS were run for 2016. The software algorithm collects the data and process the same using the DisMod-MR 2.1,( (Disease Modeling Meta-Regression Tool- 2, Free tool, Jan Barendregt, Department of Public Health of Erasmus University, Netherlands, Available from www.epigear. com). The algorithm provides with post-adjusted incidence, prevalence, mortality, and remission data( http://www.healthdata.org/data-visualization/epi-viz) after adjusting for all covariates, outliers and input data variations. The details of the model assumptions, priori, beta and the model flow chart can be accessed elsewhere. $^{15}$ 
Prevalence were the estimate number of patients in each MISUDs while the incidence (expressed as a rate) for this study purpose refers to the number of new cases per person-year. It is often approximated by taking the number of new cases in a year divided by the mid-year population of India as estimated by the population figures based on World Population Prospects: 2015 Revision, from the United Nations Population Division, and the WHO Human Mortality Database (16970 lakh males and 6527 lakh females). For the purpose of this study, remission (expressed as a rate) is the number of cases that resolve or are cured per person-year. Person-year refers to the estimate of the actual time-at-risk in years that all persons contributed to a diseases, specific MISUD while rate is the number of new cases of MISUD during a period of time, (2016 in this study), divided by the person-time-at-risk.

The GBD defines remission as no longer fulfulling the diagnostic criteria for the disorder.Remission was reported as defined as an annual hazard rate to recover from the disease, with person-time with disease in the denominator. The time factor was not stated while previous reports of a disease free state of 2-years (1-year period for major depressive disorder).

Often, these data are rarely available therefore where number of remitted cases were reported, the remission rate was calculated:

$$
\text { Weighted remission rate }(\%)=\sum\left[a * \frac{-\ln (1-b)}{c}\right] / \sum a
$$

where is sample size, $\mathrm{b}$ is remission proportion and $c$ is follow-up (years) where sufficient data were reported. ${ }^{17}$ We employed the "country final" option which represents estimate for India (blue) and "global final"(green) to compare. Global final is the final estimate fit to all the world's data after accounting for all covariates. Remission estimates from prospective general population cohort studies with a naturalistic (non-interventional) follow-up of cases until they no longer fulfilled diagnostic criteria were used and was set to predetermined prior information based on literature.

GBD Epidemiological visualization applies a $\log$ rate model of disease prevalence, incidence, remission, and case-fatality rates and fits models with a randomized Markov-Chain Monte Carlo algorithm. This model accounts for all possible outcomes and its probabilities, then incrementally performing risk analysis. The model accounts for the fixed and random factors. ${ }^{4-14,17}$ The statistical calculation model of GBD exhibits the significant effects both in co-efficient and exponentiated co-efficient, that can be considered as odds ratio. From the interactive map option, age-standardized rates of incidence and remission with $95 \%$ UI for either genders for all MISUDs listed were obtained. Using the plot option, the age-group for the remission rates for MISUDs of either gender were obtained and presented.

From the prevalence and incidence numbers derived for the Indian MISUDs for 2016, we attempted to

$$
\text { Average duration of disease }=\frac{\text { Prevalence }}{\text { Incidence }}
$$

calculate the average duration of MISUD. We assumed that that all other parameters remain same, the average treatment duration was derived using the formula ${ }^{18}$

\section{RESULTS}

In all there were 8 major data sources -7 surveys and one mixed estimation type of study that provided 1389 data points. Details of schizophrenia were acquired from 2 studies which were in the 1988-89 and 197893 time period. The other MISUDs had several data points. All the details are listed in Supplementary file-1. The definitions employed, procedural details and data processing algorithm for the study are reported in detail elsewhere. ${ }^{15}$

In 2016, 111.71 million Indians $(8.33 \%)$ suffered from the MISUDs combined, among the 1.34 billion population. Of this MIUDs, those suffering with anxiety disorders and alcohol use disorders were more prevalent. Among males, major depressive disorders predominated while among females it is the anxiety disorders that predominated. The lowest was the cocaine use disorders both in males and females (Table1 and 2). The incidence rate of age-standardized Indians in 2016 accounting for the number of new cases per person-year are listed in Table 3. Major depressive disorders emerged as the highest rate among both the genders followed by anxiety disorders. Schizophrenia, bipolar disorders and cocaine use disorders had the least incidence rate. On applying the population, in 2016, an estimated 354.86 lakh males and 370.67 lakh females, together 725.53 lakh $(5.4 \%)$ of the population had been diagnosed with MISUDs between them.

For schizophrenia and bipolar mood disorders, in males and females prevalence rate (per 100,000) was observed to be increasing till $5^{\text {th }}$ decade of life after which it reduces. In major depressive disorder the 
Table 1: Estimated prevalence of mental illness among Indians in 2016 by gender

\begin{tabular}{|l|c|c|c|c|c|c|}
\hline & \multicolumn{2}{|c|}{ Male } & \multicolumn{2}{c|}{ Female } & \multicolumn{2}{c|}{ Combined } \\
\hline $\begin{array}{l}\text { Disease/ } \\
\text { illness/ } \\
\text { Disorder }\end{array}$ & Prevalence & $95 \%$ Cl & Prevalence & 95\% CI & Prevalence & 95\% Cl \\
\hline $\begin{array}{l}\text { Schizophrenia } \\
\begin{array}{l}\text { Major } \\
\text { depressive } \\
\text { disorder }\end{array}\end{array}$ & 16.77 & $14.73-18.92$ & 14.85 & $13.05-16.71$ & 31.62 & $27.80-35.60$ \\
$\begin{array}{l}\text { Bipolar } \\
\text { disorder } \\
\begin{array}{l}\text { Anxiety } \\
\text { disorders }\end{array}\end{array}$ & 127.70 & $116.03-141.30$ & 172.49 & $156.97-190.12$ & 300.19 & $272.86-331.42$ \\
\hline
\end{tabular}

$\mathrm{Cl}$ - Confidence Interval

Table 2: Estimated prevalence of substance use disorder among Indians in 2016 by gender

\begin{tabular}{|c|c|c|c|c|c|c|}
\hline \multirow[b]{2}{*}{ Disorder } & \multicolumn{2}{|c|}{ Male } & \multicolumn{2}{|c|}{ Female } & \multicolumn{2}{|c|}{ Combined } \\
\hline & Prevalence & $95 \% \mathrm{Cl}$ & Prevalence & $95 \% \mathrm{Cl}$ & Prevalence & $95 \% \mathrm{Cl}$ \\
\hline Alcohol use & 124.35 & $111.01-139.10$ & 30,98 & $26.04-36.37$ & 155.32 & $138.51-175.10$ \\
\hline Opioid use & 23.31 & $19.99-27.18$ & 15.18 & $12.66-18.05$ & 38.48 & $32.86-45.22$ \\
\hline Cocaine use & 2.26 & $1.97-2.63$ & 0.92 & $0.78-1.11$ & 3.18 & $2.75-3.74$ \\
\hline Cannabis use & 23.21 & $19.13-28.10$ & 6.26 & $5.20-74.55$ & 29.48 & $24.51-35.30$ \\
\hline
\end{tabular}

$\mathrm{Cl}$ - Confidence Interval

Table 3: Estimated incidence rate of mental illness and substance use disorder (per Lakh) among age-standardized Indians in 2016 by gender

\begin{tabular}{|c|c|c|c|c|}
\hline \multirow[b]{2}{*}{ Disease/illness/Disorder } & \multicolumn{2}{|c|}{ Male } & \multicolumn{2}{|c|}{ Female } \\
\hline & Rate Per lakh & $\begin{array}{c}95 \% \mathrm{Cl} \\
\text { Lower-upper }\end{array}$ & Rate per Lakh & $\begin{array}{c}95 \% \mathrm{Cl} \\
\text { Lower-upper }\end{array}$ \\
\hline Schizophrenia & 14 & $12-15$ & 12 & $11-14$ \\
\hline Major depressive disorder & 3200 & $2900-3500$ & 4600 & $4100-5000$ \\
\hline Bipolar disorder & 44 & $38-50$ & 50 & $44-57$ \\
\hline Anxiety disorders & 530 & $490-560$ & 550 & $520-559$ \\
\hline Alcohol use disorders & 940 & $830-1100$ & 270 & $230-310$ \\
\hline Opioid use disorders & 31 & $26-36$ & 28 & $25-33$ \\
\hline Cocaine use disorders & 3.3 & $2.8-3.9$ & 1.8 & $1.5-2.1$ \\
\hline Cannabis use disorders & 49 & $40-59$ & 17 & $14-21$ \\
\hline
\end{tabular}

$\mathrm{Cl}$ - Confidence Interval 
Table 4: Prevalence rate of Mental Illness per 100,000 Indians by age group and gender in 2016 [95\% confidence Interval in brackets]

\begin{tabular}{|c|c|c|c|c|c|c|c|c|}
\hline \multirow{2}{*}{$\begin{array}{l}\text { Age } \\
\text { group }\end{array}$} & \multicolumn{2}{|c|}{ Schizophrenia } & \multicolumn{2}{|c|}{ Major depressive disorder } & \multicolumn{2}{|c|}{ Bipolar disorder } & \multicolumn{2}{|c|}{ Anxiety disorder } \\
\hline & Males & Females & Males & Females & Males & Females & Males & Females \\
\hline 10 to 14 & $\begin{array}{c}5 \\
{[3-8]}\end{array}$ & $\begin{array}{c}4 \\
{[2-7]}\end{array}$ & $\begin{array}{c}518 \\
{[359-719]}\end{array}$ & $\begin{array}{c}705 \\
{[492-981]}\end{array}$ & $\begin{array}{c}168 \\
{[117-226]}\end{array}$ & $\begin{array}{c}202 \\
{[141-270]}\end{array}$ & $\begin{array}{c}2471 \\
{[2154-2859]}\end{array}$ & $\begin{array}{c}3519 \\
{[3083-4073]}\end{array}$ \\
\hline 15 to 19 & $\begin{array}{c}45 \\
{[31-62]}\end{array}$ & $\begin{array}{c}40 \\
{[27-55]}\end{array}$ & $\begin{array}{c}1347 \\
{[1007-1722]}\end{array}$ & $\begin{array}{c}1858 \\
{[1412-2350]}\end{array}$ & $\begin{array}{c}572 \\
{[419-753]}\end{array}$ & $\begin{array}{c}679 \\
{[498-891]}\end{array}$ & $\begin{array}{c}2885 \\
{[2576-3242]}\end{array}$ & $\begin{array}{c}4171 \\
{[3737-4682]}\end{array}$ \\
\hline 20 to 24 & $\begin{array}{c}173 \\
{[123-228]}\end{array}$ & $\begin{array}{c}156 \\
{[111-203]}\end{array}$ & $\begin{array}{c}1842 \\
{[1418-2398]}\end{array}$ & $\begin{array}{c}2514 \\
{[1916-3265]}\end{array}$ & $\begin{array}{c}644 \\
{[502-798]}\end{array}$ & $\begin{array}{c}741 \\
{[577-919]}\end{array}$ & $\begin{array}{c}2760 \\
{[2408-3155]}\end{array}$ & $\begin{array}{c}4006 \\
{[3512-4539]}\end{array}$ \\
\hline 25 to 29 & $\begin{array}{c}310 \\
{[237-393]}\end{array}$ & $\begin{array}{c}286 \\
{[220-365]}\end{array}$ & $\begin{array}{c}1825 \\
{[1465-2241]}\end{array}$ & $\begin{array}{c}2603 \\
{[2087-3202]}\end{array}$ & $\begin{array}{c}641 \\
{[511-798]}\end{array}$ & $\begin{array}{c}721 \\
{[575-898]}\end{array}$ & $\begin{array}{c}2843 \\
{[2484-3237]}\end{array}$ & $\begin{array}{c}4108 \\
{[3589-4692]}\end{array}$ \\
\hline 30 to 34 & $\begin{array}{c}410 \\
{[334-492]}\end{array}$ & $\begin{array}{c}383 \\
{[310-458]}\end{array}$ & $\begin{array}{c}1991 \\
{[1544-2520]}\end{array}$ & $\begin{array}{c}2875 \\
{[2220-3618]}\end{array}$ & $\begin{array}{c}651 \\
{[526-802]}\end{array}$ & $\begin{array}{c}726 \\
{[584-892]}\end{array}$ & $\begin{array}{c}3101 \\
{[2814-3423]}\end{array}$ & $\begin{array}{c}4482 \\
{[4016-4996]}\end{array}$ \\
\hline 35 to 39 & $\begin{array}{c}471 \\
{[393-555]}\end{array}$ & $\begin{array}{c}442 \\
{[369-519]}\end{array}$ & $\begin{array}{c}2529 \\
{[2064-3045]}\end{array}$ & $\begin{array}{c}3552 \\
{[2918-4242]}\end{array}$ & $\begin{array}{c}674 \\
{[545-820]}\end{array}$ & $\begin{array}{c}751 \\
{[611-917]}\end{array}$ & $\begin{array}{c}3425 \\
{[3102-3772]}\end{array}$ & $\begin{array}{c}5036 \\
{[4554-5526]}\end{array}$ \\
\hline 40 to 44 & $\begin{array}{c}500 \\
{[427-575]}\end{array}$ & $\begin{array}{c}470 \\
{[404-540]}\end{array}$ & $\begin{array}{c}2949 \\
{[2337-3585]}\end{array}$ & $\begin{array}{c}4083 \\
{[3266-4955]}\end{array}$ & $\begin{array}{c}701 \\
{[554-862]}\end{array}$ & $\begin{array}{c}783 \\
{[621-960]}\end{array}$ & $\begin{array}{c}3710 \\
{[3301-4150]}\end{array}$ & $\begin{array}{c}5577 \\
{[5011-6142]}\end{array}$ \\
\hline 45 to 49 & $\begin{array}{c}509 \\
{[443-581]}\end{array}$ & $\begin{array}{c}478 \\
{[415-547]}\end{array}$ & $\begin{array}{c}3149 \\
{[2676-3647]}\end{array}$ & $\begin{array}{c}4409 \\
{[3751-5080]}\end{array}$ & $\begin{array}{c}710 \\
{[567-861]}\end{array}$ & $\begin{array}{c}793 \\
{[638-961]}\end{array}$ & $\begin{array}{c}3765 \\
{[3383-4187]}\end{array}$ & $\begin{array}{c}5704 \\
{[5153-6315]}\end{array}$ \\
\hline 50 to 54 & $\begin{array}{c}501 \\
{[438-574]}\end{array}$ & $\begin{array}{c}471 \\
{[412-539]}\end{array}$ & $\begin{array}{c}3329 \\
{[2875-3802]}\end{array}$ & $\begin{array}{c}4729 \\
{[4089-5427]}\end{array}$ & $\begin{array}{c}698 \\
{[564-853]}\end{array}$ & $\begin{array}{c}780 \\
{[635-956]}\end{array}$ & $\begin{array}{c}3682 \\
{[3355-4042]}\end{array}$ & $\begin{array}{c}5504 \\
{[5026-6021]}\end{array}$ \\
\hline 55 to 59 & $\begin{array}{c}480 \\
{[422-545]}\end{array}$ & $\begin{array}{c}450 \\
{[396-510]}\end{array}$ & $\begin{array}{c}3531 \\
{[2934-4176]}\end{array}$ & $\begin{array}{c}5016 \\
{[4185-5930]}\end{array}$ & $\begin{array}{c}666 \\
{[543-814]}\end{array}$ & $\begin{array}{c}748 \\
{[617-916]}\end{array}$ & $\begin{array}{c}3543 \\
{[3217-3913]}\end{array}$ & $\begin{array}{c}5077 \\
{[4639-5557]}\end{array}$ \\
\hline 60 to 64 & $\begin{array}{c}442 \\
{[388-499]}\end{array}$ & $\begin{array}{c}414 \\
{[365-466]}\end{array}$ & $\begin{array}{c}3764 \\
{[3132-4470]}\end{array}$ & $\begin{array}{c}5460 \\
{[4575-6456]}\end{array}$ & $\begin{array}{c}617 \\
{[514-744]}\end{array}$ & $\begin{array}{c}699 \\
{[583-841]}\end{array}$ & $\begin{array}{c}3363 \\
{[2961-3840]}\end{array}$ & $\begin{array}{c}4594 \\
{[4122-5116]}\end{array}$ \\
\hline 65 to 69 & $\begin{array}{c}375 \\
{[331-423]}\end{array}$ & $\begin{array}{c}352 \\
{[309-397]}\end{array}$ & $\begin{array}{c}4171 \\
{[3656-4791]}\end{array}$ & $\begin{array}{c}6041 \\
{[5334-6874]}\end{array}$ & $\begin{array}{c}554 \\
{[456-677]}\end{array}$ & $\begin{array}{c}630 \\
{[516-768]}\end{array}$ & $\begin{array}{c}3211 \\
{[2835-3667]}\end{array}$ & $\begin{array}{c}4341 \\
{[3834-4892]}\end{array}$ \\
\hline 70 to 74 & $\begin{array}{c}290 \\
{[255-327]}\end{array}$ & $\begin{array}{c}273 \\
{[240-310]}\end{array}$ & $\begin{array}{c}4514 \\
{[3906-5203]}\end{array}$ & $\begin{array}{c}6551 \\
{[5773-7464]}\end{array}$ & $\begin{array}{c}483 \\
{[396-587]}\end{array}$ & $\begin{array}{c}548 \\
{[450-665]}\end{array}$ & $\begin{array}{c}3062 \\
{[2757-3423]}\end{array}$ & $\begin{array}{c}4288 \\
{[3850-4811]}\end{array}$ \\
\hline 75 to 79 & $\begin{array}{c}203 \\
{[176-231]}\end{array}$ & $\begin{array}{c}193 \\
{[167-220]}\end{array}$ & $\begin{array}{c}4894 \\
{[4021-5840]}\end{array}$ & $\begin{array}{c}7045 \\
{[5935-8269]}\end{array}$ & $\begin{array}{c}412 \\
{[339-497]}\end{array}$ & $\begin{array}{c}465 \\
{[385-559]}\end{array}$ & $\begin{array}{c}2935 \\
{[2669-3240]}\end{array}$ & $\begin{array}{c}4375 \\
{[3977-4823]}\end{array}$ \\
\hline 80 plus & $\begin{array}{c}107 \\
{[90-126]}\end{array}$ & $\begin{array}{c}102 \\
{[86-121]}\end{array}$ & $\begin{array}{c}4653 \\
{[3870-5498]}\end{array}$ & $\begin{array}{c}6470 \\
{[5487-7557]}\end{array}$ & $\begin{array}{c}327 \\
{[266-400]}\end{array}$ & $\begin{array}{c}363 \\
{[297-441]}\end{array}$ & $\begin{array}{c}2617 \\
{[2345-2939]}\end{array}$ & $\begin{array}{c}4339 \\
{[3935-4822]}\end{array}$ \\
\hline
\end{tabular}

prevalence rates progressively increased with age in both gender. For the anxiety disorder, prevalence rates was highest in 45 to 49 years in males and females after which it reduces (Table 4).

Alcohol use disorder prevalence rates was highest in 30 to 34 years age group among males and females after which it decreased. For opioid disorder, highest prevalence rate for males was in 30 to 34 years age group while for females it is 25 to 29 years age group. Cocaine use disorder was more prevalent in $3^{\text {rd }}$ decade of life in both gender while 20 to 24 years age group showed highest prevalence rates for cannabis use disorder (Table 4).

Age standardized, remission expressed as a rate, refers to the number of cases that resolve or are cured per person-year, for the year 2016 are given in Table 5. Major depressive disorders showed high remission rate while schizophrenia and bipolar disorders show the least rate. Among substances, alcohol use disorder rates were the highest followed by opioid while other remained the least. The model showed that gender was a significant covariate for alcohol and opioid use disorders, anxiety disorders and conduct disorders. The fixed effect values of the $\mathrm{x}$-covariatein this model (which means that data points are adjusted to the reference value if a systematic bias is detected) which are in log space (as DisMod uses an offset lognormal model) as well as the exponentiated values which for an $\mathrm{x}$-covariates can be interpreted as an odds ratio. ${ }^{17}$ The beta and the exponentiated beta are given in Table 5 along with 95\% UI. The random effects were not observed for any of the MISUDs studied.

The effect of age on the remission rate were studied in detail. Each MISUD had a distinct age based remission as evidenced in the Figures 1 and 2. Except 
Table 5: Prevalence rate of Substance use disorder per 100,000 Indians by age group and gender in 2016 [95\% Confidence Interval, in brackets]

\begin{tabular}{|c|c|c|c|c|c|c|c|c|}
\hline \multirow{2}{*}{$\begin{array}{l}\text { Age } \\
\text { group }\end{array}$} & \multicolumn{2}{|c|}{ Alcohol use disorder } & \multicolumn{2}{|c|}{ Opioid use disorder } & \multicolumn{2}{|c|}{ Cocaine use disorder } & \multicolumn{2}{|c|}{ Cannabis use disorder } \\
\hline & Males & Females & Males & Females & Males & Females & Males & Females \\
\hline 10 to 14 & $\begin{array}{c}69 \\
{[41-111]}\end{array}$ & $\begin{array}{c}32 \\
{[18-54]}\end{array}$ & 0 & 0 & 0 & 0 & $\begin{array}{c}39 \\
{[10-76]}\end{array}$ & $\begin{array}{c}7 \\
{[1-17]}\end{array}$ \\
\hline 15 to 19 & $\begin{array}{c}736 \\
{[530-980]}\end{array}$ & $\begin{array}{c}277 \\
{[185-396]}\end{array}$ & $\begin{array}{c}97 \\
{[75-126]}\end{array}$ & $\begin{array}{c}82 \\
{[61-110]}\end{array}$ & $\begin{array}{c}34 \\
{[22-50]}\end{array}$ & $\begin{array}{c}16 \\
{[9-25]}\end{array}$ & $\begin{array}{c}622 \\
{[387-914]}\end{array}$ & $\begin{array}{c}157 \\
{[104-234]}\end{array}$ \\
\hline 20 to 24 & $\begin{array}{c}2107 \\
{[1564-2761]}\end{array}$ & $\begin{array}{c}664 \\
{[446-938]}\end{array}$ & $\begin{array}{c}520 \\
{[403-662]}\end{array}$ & $\begin{array}{c}426 \\
{[322-565]}\end{array}$ & $\begin{array}{c}56 \\
{[44-72]}\end{array}$ & $\begin{array}{c}28 \\
{[21-37]}\end{array}$ & $\begin{array}{c}1022 \\
{[748-1352]}\end{array}$ & $\begin{array}{c}312 \\
\text { [229-412] }\end{array}$ \\
\hline 25 to 29 & $\begin{array}{c}3084 \\
{[2515-3765]}\end{array}$ & $\begin{array}{c}791 \\
\text { [588-1062] }\end{array}$ & $\begin{array}{c}837 \\
{[678-1036]}\end{array}$ & $\begin{array}{c}626 \\
{[495-798]}\end{array}$ & $\begin{array}{c}58 \\
{[47-73]}\end{array}$ & $\begin{array}{c}29 \\
{[22-37]}\end{array}$ & $\begin{array}{c}762 \\
{[560-1028]}\end{array}$ & $\begin{array}{c}228 \\
{[167-305]}\end{array}$ \\
\hline 30 to 34 & $\begin{array}{c}3547 \\
{[2870-4335]}\end{array}$ & $\begin{array}{c}829 \\
{[590-1152]}\end{array}$ & $\begin{array}{c}890 \\
{[709-1084]}\end{array}$ & $\begin{array}{c}578 \\
{[437-727]}\end{array}$ & $\begin{array}{c}52 \\
{[40-66]}\end{array}$ & $\begin{array}{c}24 \\
{[18-32]}\end{array}$ & $\begin{array}{c}518 \\
{[390-676]}\end{array}$ & $\begin{array}{c}149 \\
{[111-194]}\end{array}$ \\
\hline 35 to 39 & $\begin{array}{c}3427 \\
{[2823-4084]}\end{array}$ & $\begin{array}{c}821 \\
\text { [621-1081] }\end{array}$ & $\begin{array}{c}705 \\
{[563-857]}\end{array}$ & $\begin{array}{c}433 \\
{[335-547]}\end{array}$ & $\begin{array}{c}47 \\
{[38-59]}\end{array}$ & $\begin{array}{c}20 \\
{[15-26]}\end{array}$ & $\begin{array}{c}384 \\
{[284-502]}\end{array}$ & $\begin{array}{c}113 \\
{[82-153]}\end{array}$ \\
\hline 40 to 44 & $\begin{array}{c}3210 \\
{[2480-4001]}\end{array}$ & $\begin{array}{c}809 \\
{[554-1168]}\end{array}$ & $\begin{array}{c}461 \\
{[364-577]}\end{array}$ & $\begin{array}{c}292 \\
{[227-369]}\end{array}$ & $\begin{array}{c}44 \\
{[36-55]}\end{array}$ & $\begin{array}{c}18 \\
{[13-23]}\end{array}$ & $\begin{array}{c}291 \\
{[214-376]}\end{array}$ & $\begin{array}{c}87 \\
{[63-115]}\end{array}$ \\
\hline 45 to 49 & $\begin{array}{c}2978 \\
{[2448-3580]}\end{array}$ & $\begin{array}{c}793 \\
{[603-1035]}\end{array}$ & $\begin{array}{c}327 \\
{[261-407]}\end{array}$ & $\begin{array}{c}218 \\
{[173-279]}\end{array}$ & $\begin{array}{c}42 \\
{[34-52]}\end{array}$ & $\begin{array}{c}16 \\
{[12-21]}\end{array}$ & $\begin{array}{c}240 \\
{[182-313]}\end{array}$ & $\begin{array}{c}74 \\
{[56-94]}\end{array}$ \\
\hline 50 to 54 & $\begin{array}{c}2728 \\
{[2074-3407]}\end{array}$ & $\begin{array}{c}763 \\
{[540-1014]}\end{array}$ & $\begin{array}{c}270 \\
{[211-330]}\end{array}$ & $\begin{array}{c}188 \\
{[145-240]}\end{array}$ & $\begin{array}{c}41 \\
{[33-50]}\end{array}$ & $\begin{array}{c}15 \\
{[11-19]}\end{array}$ & $\begin{array}{c}165 \\
{[128-213]}\end{array}$ & $\begin{array}{c}49 \\
{[37-65]}\end{array}$ \\
\hline 55 to 59 & $\begin{array}{c}2465 \\
{[2007-2998]}\end{array}$ & $\begin{array}{c}690 \\
{[532-879]}\end{array}$ & $\begin{array}{c}257 \\
{[207-312]}\end{array}$ & $\begin{array}{c}185 \\
{[148-229]}\end{array}$ & $\begin{array}{c}42 \\
{[34-50]}\end{array}$ & $\begin{array}{c}15 \\
{[11-19]}\end{array}$ & $\begin{array}{c}104 \\
{[79-135]}\end{array}$ & $\begin{array}{c}36 \\
{[27-46]}\end{array}$ \\
\hline 60 to 64 & $\begin{array}{c}2215 \\
{[1693-2791]}\end{array}$ & $\begin{array}{c}597 \\
{[411-825]}\end{array}$ & $\begin{array}{c}260 \\
{[226-298]}\end{array}$ & $\begin{array}{c}189 \\
{[158-225]}\end{array}$ & $\begin{array}{c}44 \\
{[37-51]}\end{array}$ & $\begin{array}{c}15 \\
{[12-18]}\end{array}$ & $\begin{array}{c}67 \\
{[54-83]}\end{array}$ & $\begin{array}{c}31 \\
{[24-38]}\end{array}$ \\
\hline 65 to 69 & $\begin{array}{c}1986 \\
{[1592-2443]}\end{array}$ & $\begin{array}{c}469 \\
{[346-604]}\end{array}$ & $\begin{array}{c}247 \\
{[213-284]}\end{array}$ & $\begin{array}{c}170 \\
{[141-204]}\end{array}$ & $\begin{array}{c}42 \\
{[35-49]}\end{array}$ & $\begin{array}{c}14 \\
{[11-18]}\end{array}$ & $\begin{array}{c}52 \\
{[41-65]}\end{array}$ & $\begin{array}{c}28 \\
{[21-35]}\end{array}$ \\
\hline 70 to 74 & $\begin{array}{c}1717 \\
{[1348-2181]}\end{array}$ & $\begin{array}{c}360 \\
{[258-493]}\end{array}$ & $\begin{array}{c}218 \\
{[187-251]}\end{array}$ & $\begin{array}{c}132 \\
{[111-156]}\end{array}$ & $\begin{array}{c}37 \\
{[31-43]}\end{array}$ & $\begin{array}{c}13 \\
{[10-16]}\end{array}$ & $\begin{array}{c}44 \\
{[36-55]}\end{array}$ & $\begin{array}{c}24 \\
{[18-31]}\end{array}$ \\
\hline 75 to 79 & $\begin{array}{c}1369 \\
{[1116-1677]}\end{array}$ & $\begin{array}{c}322 \\
{[240-416]}\end{array}$ & $\begin{array}{c}195 \\
{[168-225]}\end{array}$ & $\begin{array}{c}106 \\
{[89-126]}\end{array}$ & $\begin{array}{c}32 \\
{[27-38]}\end{array}$ & $\begin{array}{c}11 \\
{[9-14]}\end{array}$ & $\begin{array}{c}40 \\
{[31-49]}\end{array}$ & $\begin{array}{c}22 \\
{[16-28]}\end{array}$ \\
\hline 80 plus & $\begin{array}{c}1101 \\
{[887-1350]}\end{array}$ & $\begin{array}{c}344 \\
{[270-433]}\end{array}$ & $\begin{array}{c}170 \\
{[145-199]}\end{array}$ & $\begin{array}{c}86 \\
{[71-104]}\end{array}$ & $\begin{array}{c}27 \\
{[23-32]}\end{array}$ & $\begin{array}{c}10 \\
{[7-12]}\end{array}$ & $\begin{array}{c}33 \\
{[26-41]}\end{array}$ & $\begin{array}{c}18 \\
{[13-23]}\end{array}$ \\
\hline
\end{tabular}

for schizophrenia and major depressive disorders, age appears to be influencing the remission characteristics.

As seen in Figure1, in males, for alcohol dependence, the remission rate appears to increase till age of 40 years after which it decreases.Among cannabis use disorder patients, remission increases till early 20s after which it is not steady and again peaks by 60 years, after which it decreases by 80 and the again increases. For cocaine use disorders, the remission rates increases till 20 years after which it slowly reduces while for opioid disorder, the remission rate peaks by age of early 40 after which it declines. For major depressive disorder, the remission rate is nearly stable around 1.5 cases per person-year. There is a similar trend between the global and Indian trends. For schizophrenia, the global trend is higher than Indians but is nearly constant across age group. For females, the trend is the same as the males but with slightly different remission rates (Figure 2). For all MISUDs, the remission rate was well below the upper limit and priori, indicating the cure rate were below the inputs as cited in the model inputs. ${ }^{15}$ The model beta and the exponentiated beta, (equivalent to odds ratio) are given in Table 6 . The random and fixed effects for any other covariates emerged significant for any of the MISUDs studied. When male was taken as reference category, odds ratio 0.59 shows that female had $41 \%$ reduced risk of alcohol use disorder compared to male. Similar trend noted for opioid disorders. Females are twice prone for anxiety disorders female as compared to males.

In males, the average duration of disease was highest with schizophrenia (16.92 years) followed by bipolar disorders (10.41 years) while least was with major depressive disorder ( 0.65 years). Females followed the duration trend but with difference. For Schizophrenia, bipolar disorder and anxiety disorder, females had a longer average duration than the males while for others males had longer duration of diseases/disorder (Table 7). 
Figure 1: Graph depicting the combined, collective effect of age on the remission rate among males in the studied conditions.

A. Alcohol Dependence B. Anxiety Disorder Cases C. Bipolar disorder cases D. Cannabis use disorder E. Cocaine Use disorder F. Major Depressive disorders G. Opioid Use disorders H. Schizophrenia

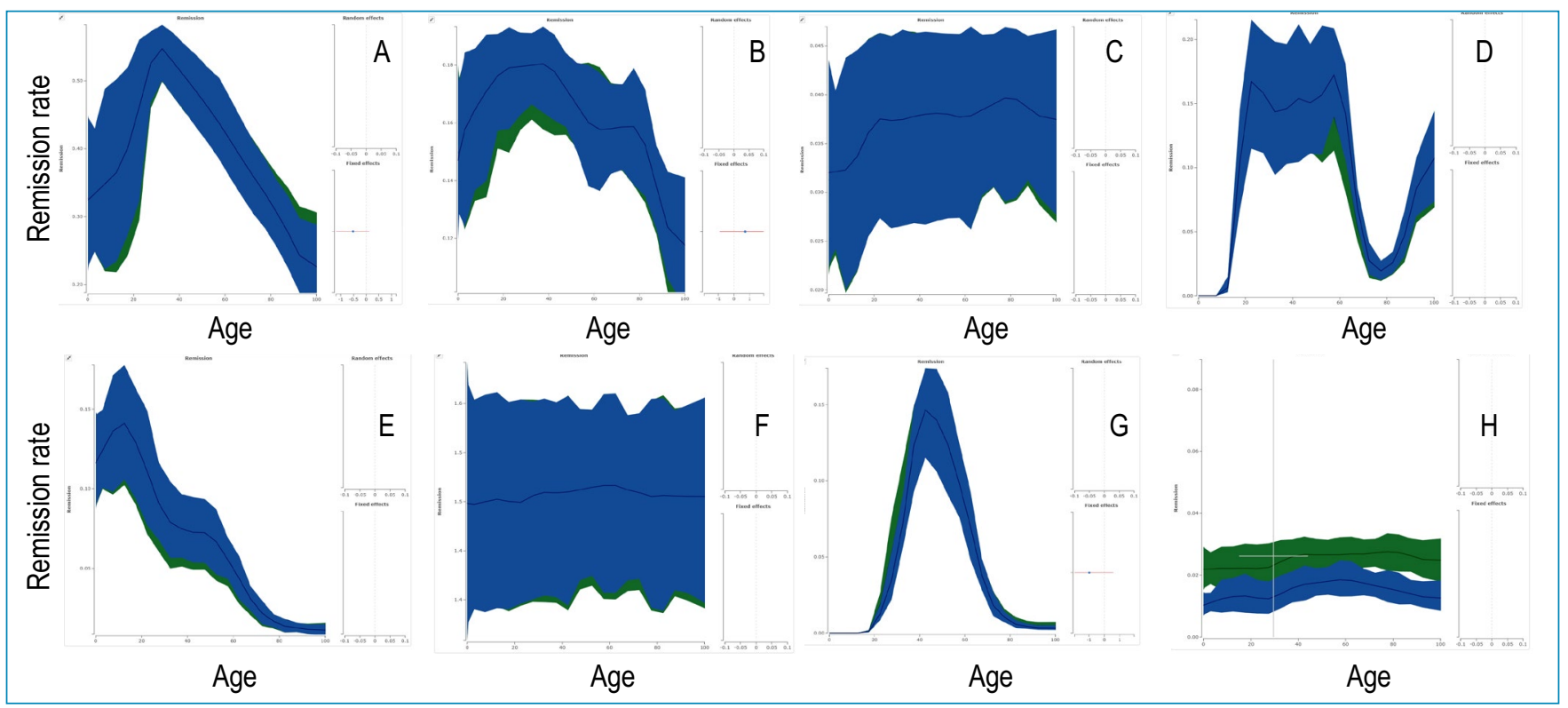

Figure 2: Graph depicting the combined, collective effect of age on the remission rate among females in the studied conditions

A. Alcohol Dependence B. Anxiety Disorder Cases C. Bipolar disorder cases D. Cannabis use disorder E. Cocaine Use disorder F. Major Depressive disorders G. Opioid Use disorders H. Schizophrenia
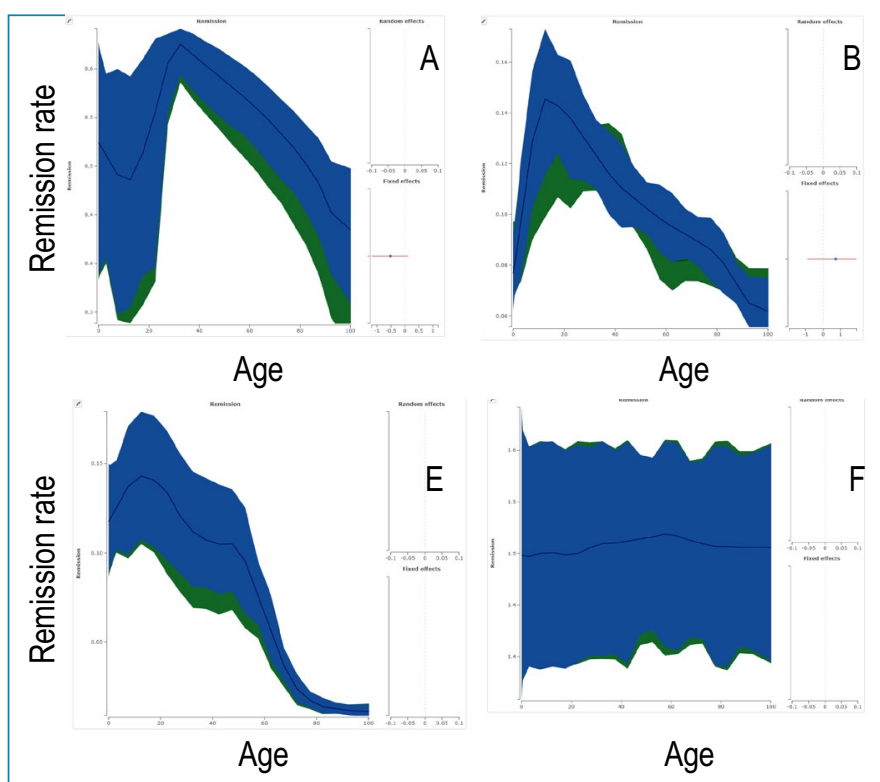

Age
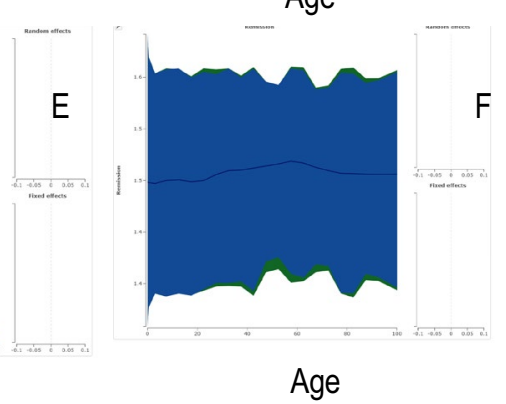

Age

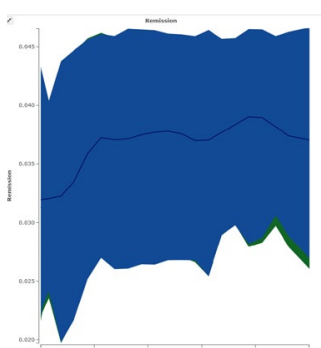

Ầge

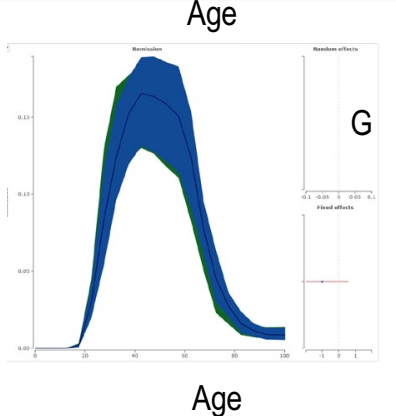

Age

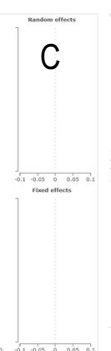

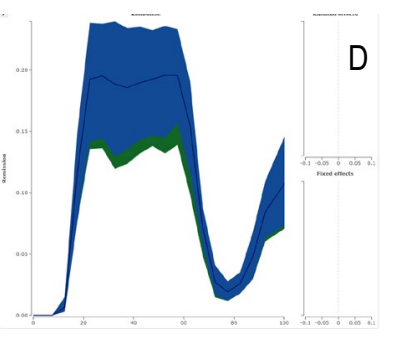

Age

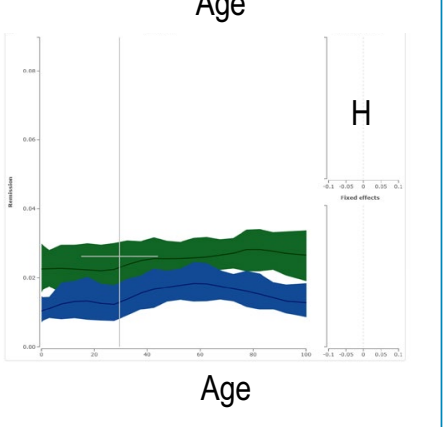

\section{DISCUSSION}

Given the diverse nature of outcome of MISUDs, the goal of any related therapy would be to shun substances in substance use disorders and return to wellness for other disorders. ${ }^{19}$ It is a common fact that for serious chronic disorders patients are often under continuous medical observation and outcomes

are often documented. In chronic episodic disorders, the existing health monitoring systems only partially capture the outcome, as when a remission occurs. In remission states, patients do not report or seek medical help and thus remain out of medical surveillance. Remission focused studies offer a chance to introspect the standard of care, clinical evidence of treatment and 
Table 6: Remission (expressed as rate) of mental illness and substance use disorder among age-standardized Indians in 2016 by Gender and model effects

\begin{tabular}{|c|c|c|c|c|c|c|c|c|c|}
\hline \multirow{3}{*}{$\begin{array}{c}\text { Disease /illness/ } \\
\text { Disorder }\end{array}$} & \multirow{3}{*}{$\begin{array}{l}\text { Upper } \\
\text { limit of } \\
\text { remission } \\
\text { rate set in } \\
\text { the model }\end{array}$} & \multicolumn{2}{|c|}{$\begin{array}{c}\text { Remission Rate-Number of cases that resolve } \\
\text { or are cured per person-year ( } 95 \% \text { Confidence } \\
\text { Interval) }\end{array}$} & \multicolumn{6}{|c|}{ Fixed effect } \\
\hline & & \multirow{2}{*}{ Male } & \multirow{2}{*}{ Female } & \multicolumn{2}{|c|}{ Beta } & \multicolumn{3}{|c|}{ Exponentiated Beta } & \multirow[b]{2}{*}{ Higher } \\
\hline & & & & Value & Lower & Higher & Value & Lower & \\
\hline Schizophrenia & 0.04 & $0.015(0.013-0.017)$ & $0.015(0.012-0.017)$ & & & & & & \\
\hline $\begin{array}{l}\text { Alcohol use } \\
\text { disorders }\end{array}$ & 0.6 & $0.43(0.39-0.47)$ & $0.50(0.47-0.54)$ & -0.52 & -1.19 & 0.12 & 0.59 & 0.3 & 1.13 \\
\hline Opioid use disorders & 0.2 & $0.051(0.045-0.058)$ & $0.073(0.064-0.083)$ & -0.98 & -1.94 & 0.59 & 0.38 & 0.14 & 1.81 \\
\hline $\begin{array}{l}\text { Cocaine use } \\
\text { disorders }\end{array}$ & 0.2 & $0.091(0.08-0.1)$ & $0.11(0.096-0.12)$ & & & & & & \\
\hline $\begin{array}{l}\text { Cannabis use } \\
\text { disorders }\end{array}$ & 0.2 & $0.099(0.089-0.11)$ & $0.12(0.11-0.13)$ & & & & & & \\
\hline $\begin{array}{l}\text { Major depressive } \\
\text { disorder }^{\$}\end{array}$ & 1.45 & $1.46(1.41-1.5)$ & $1.46(1.41-1.5)$ & & & & & & \\
\hline Bipolar disorder & 0.05 & $0.036(0.032-0.041)$ & $0.036(0.032-0.04)$ & & & & & & \\
\hline Anxiety disorders & 0.2 & $0.17(0.16-0.18)$ & $0.12(0.11-0.12)$ & 0.72 & -0.91 & 1.91 & 2.05 & -4 & 6.78 \\
\hline
\end{tabular}

*based on available literature as cited in 15, supplementary appendix, pages 400-466; Remission rate is for 2-years; $\$$

Remission rate for 1-year

Table 7: Average duration of mental illness and substance use disorder (in years) among age-standardized Indians in 2016 by gender

\begin{tabular}{|l|c|c|c|c|c|c|}
\hline \multirow{2}{*}{$\begin{array}{l}\text { Disease/illness/ } \\
\text { Disorder }\end{array}$} & \multicolumn{3}{|c|}{ Male } & \multicolumn{2}{c|}{ Female } & \multicolumn{2}{c|}{ Average duration of disease } \\
In years
\end{tabular}

efficiency of the medical system. Such studies require a standardized, prospective approach of long term nature to capture remission rates. ${ }^{3}$ Analysis of such data would help to define the temporal criteria (period of time without an exacerbation of symptoms) for remission. ${ }^{16}$ Studies fulfilling such criteria are rare and existing one are often reported to be filled with logistical and methodological challenges. ${ }^{3,16}$ Mathematical methods have evolved to effectively convert cross-sectional survey data to prospective studies data to identify the remission rates. ${ }^{3,15}$

Global Burden of Diseases approach have been effectively used in the past to describe the epidemiology of the considered MISUDs. ${ }^{4-15}$ The prevalence of 
MISUDs is in tune with the projection in literature ${ }^{1}$ as the same data has been taken for the calculation of GBD. Though the remission rate, a different rate of priori taken as seen in Table 5, the resultant remission rates for schizophrenia, bipolar disorders and opioid use disorders obtained at the end of the study were lesser than this rate, indicating poor remission indicating well below the global parameters (Table 5). ${ }^{15}$ The diagnosis of remission was based on not meeting criteria for the disorders (excluding craving) while there might be elements of remnants of the disorders that is not severe enough to be called as the MISUDs but still is not normal. ${ }^{4-14,17}$ The definitions employed for the various studies are ambiguous and needs to be standardized. ${ }^{17}$ Clinical studies with therapeutic interventions quote clinical remission in the range of $30 \%$ and above for 1 -year period while complete remission is nearly unachievable in severe cases of schizophrenia, depressive disorders, bipolar disorders, anxiety and conduct disorders. While near normalcy can be achieved in substance use disorders, the rate of relapse might be high. ${ }^{19-21}$ In addition, "somatization" and non-availability of mental health professionals are other issues in primary care setting often leading to misdiagnosis and thus mismanagement. ${ }^{16}$ Spontaneous remission, regression of less severe cases to non-symptomatic state with pharmacotherapies and psychotherapies are often left behind from the watch of the existing medical system. ${ }^{2,16}$ A large proportion of such borderline cases, if kept out of the monitoring system, may cumulatively lead to larger burden of MISUDs in future. ${ }^{1}$ The lower remission rate even in achievable conditions like substance abuse disorders indicate that adequate steps needs to be taken on priority basis. The present results clearly highlights that India needs better model of mental health care delivery system than existing model as it fails to detect substantial remission or document the extent of the success.

For alcohol dependence, the remission rate appears to be rapidly increasing till age of 40 years after which it decreases. The reason for this phenomenon, especially increasing co-morbidity with chronic alcohol use leading to early, preventable mortality might be one of the reason for the sudden drop in remission rate. Institution of proper, early interventions might ensure that patients quit alcohol. ${ }^{4}$ For cannabis use disorder patients, remission rates increases till early 20s after which it plateau to peak again by seventh decade of life. This indicates that early treatment would be beneficial while latter treatment needs continuous follow-up. ${ }^{7}$ The other substance use disorders such as cocaine and opioid also exhibit such age dependent phenomenon. These may be closely related to age of initiation, strong cultural and societal reasons. ${ }^{10,12,14}$ Harnessing the age related remission rates might yield better clinical results. Depression and alcoholic use disorder that has high differential mortality rates, does not show this trend. For major depressive disorder, the remission rate is nearly stable around 1.5 cases per person-year ${ }^{9}$, which is indicative that either there are several remissions over the person-year or there is remission achieved, nearly in all the newly diagnosed cases, increasing the remission rates or there is a shorter duration of rate of recovery. Results from Table 5 further add strength to this opine. With increasing age about fifthth decade, alcohol dependence disorder, anxiety disorders and cannabis/cocaine/opioid use disorder show decreasing remission rates with age. This shows that with age, either due to disease progression or increase of comorbidities makes the remission rates lower and in agreement with previous literature and prevalence rate trend observed in Table 3 and $4 .^{2}$ All MISUDs except schizophrenia, trend of remission rates between the global and Indian trends were similar. ${ }^{2}$ For schizophrenia, the global trend is higher than Indians but is nearly constant across age group. These findings are consistent, at least in trend with reported medical literature. ${ }^{21}$

Table 6, reflects a hypothetical average length of the MISUDs on presumption that all other factors are same. Table 6 proceeds to reflect the fact of progression and is in agreement of the time reported for cure in literature. ${ }^{2,4-14,19-21}$ The difference in length of treatment between genders add strength to the influence of gender on treatment of MISUDs.

It is reported that remission along with it brings understanding of stigma, self-stigma, loss to regular medical follow-up, medication discontinuation in patients and reduction in quality of life. ${ }^{22}$ Though the extent of impact of these factors varies with MISUDs and dependent on several other factors, they cannot be taken lightly as these may trigger or predispose to other forms of disorders or even relapse. With a substantial number of Indian MISUD population not having access to mental health facility, proper employment, source of income or covered by health insurance, the low rate of remission will increase the burden. ${ }^{23,24}$ Harnessing the digital model of continuous, human-free monitoring using the power of technology via biofeedback mechanism and using smart phones or such ubiquitous devices may provide vital clue for remission and long term follow-up. 
Though sounding improbable, the prototypes has shown promising results. ${ }^{25}$ Policy makers needs to utilize the GBD data to draw meaningful conclusions to combat mental illness and substance use disorder. Indian health care providers need to identify the common socio-cultural-religious factors that might be a risk factor for mental illness including abuse, poverty, restricted or shared resources etc. As age and gender emerge as sensitive and restrictive parameters, the following recommendations are made (1) mental health screening for all adolescents is essential. Vulnerable and at risk population should be identified and properly followed up (2) gender sensitization and increasing women workforce in mental health set up needs to be done.

In 2013, an analysis of the existing mental health literatures (1998-2008) on mental health revealed that most of the data pertaining to remission comes from high-income (according to World Bank criteria) nations and other nations suffer from non-availability of data. ${ }^{17}$ They report that low prioritization of research in mental disorders, variations in study methodology impairing accuracy of epidemiological measures including case definitions, sampling, reporting and methodological barriers. They also underlined the difficulty of researchers, especially for publishing in peer-reviewed mental health journals. Language difficulties and less training prevented researchers in accessing overseas journals while poor reach and nonindexing hampered regional or national publications in non-high-income group of countries. ${ }^{17}$ The GBD 2016 study credited the Indian medical system for sharing voluminous data that led to changes in several parameters and outcome. ${ }^{15}$ In spite of these valiant efforts, still only one of the Indian psychiatrical articles found place in the data input for this study. Changes in Indian MISUDs research mindset and publication focus re-orientation, as advocated earlier is the need of the hour. ${ }^{26,27}$

The present study could suffer from biases emanating from poor data representativeness index. ${ }^{13}$ Though the DisMod II MR system has inbuilt mechanism to counteract this, the effectiveness of which is unknown. The present study relies on less number of previous publication studies/surveys for data points, especially for schizophrenia. Hence several data inputs and estimates in many instances were imputed through modelling, there by the final estimates may reflect a broad range of uncertainty. Hence the results of this study needs to be interpreted with caution..$^{4-14}$ Spontaneous remissions, those Indian who are not covered by existing medical care, especially when the treatment gap is reportedly large is a cause of concern as these may alter the projected remission trends of MISUDs. ${ }^{16}$ The present exercise to describe the prevalence remission characteristics cannot be considered futile as it has been successful in underlining the present state of Indian MISUD literature evidence and or poor mental health documentation. GBD evokes a valiant effort to predict the remission rates, an important tool for assessment of treatment as well as the outcome of MISUD care delivery. Future studies, especially at subnational level, as predicted in recent reports will highlight the more in to the prevalence, remission and MISUD care across India. ${ }^{15}$

\section{CONCLUSION}

A robust estimate of the prevalence and remission characteristics, influence of age and gender of MISUD has been presented. Till several detailed prospective study of longer duration emerges from across India to clarify the natural history of MISUD or effectiveness of treated cases of MISUDs, the results of this study would help the policy framers and clinicians to plan their community level strategy to combat mental illness and achieve the goal of wellness for their patients.

\section{ACKNOWLEDGEMENT}

Author would like to acknowledge the University of Washington and Global Burden of Diseases Colloborators and Prof. Rooban Thavarajah of Marundeeswara Oral Pathology Services and Analytics, Chennai for his critical inputs.

\section{CONFLICTS OF INTEREST}

None

\section{References}

1. Gururaj G, Varghese M, Benegal V, Rao GN, Pathak K, Singh LK, et al. National Mental Health Survey of India, 2015-16: Summary. Bengaluru: National Institute of Mental Health and Neuro Sciences, NIMHANS Publication No. 128; 2016, Bengalaru, $1^{\text {st }}$ Edition.

2. Bland RC, Newman SC, Orn H. Age and remission of psychiatric disorders. Can J Psychiatry 1997;42:722-9.

3. Roy J, Stewart WF. Methods for estimating remission rates from cross-sectional survey data: Application and validation using data from a national migraine study. Am J Epidemiol. 2011;173:949-55.

4. Rehm J, Mathers C, Popova S, Thavancharoensap M, Teerwattanaanon Y, Patra J. Global burden of disease and injury and economic cost attributable to alcohol use and alcohol-use disorders. Lancet. 2009; 373:2223-33.

5. Baxter AJ, Vos T, Scott KM, Ferrari AJ, Whiteford HA. The global burden of anxiety disorders in 2010. Psychol Med 2014;44:2363-74. 
6. Ferrari AJ, Stockings E, Khoo J, Erskine HE, Degenhardt L, Vos T, Whiteford HA. The prevalence and burden of bipolar disorder: findings from the Global Burden of Disease Study 2013. Bipolar Disorders. 2016;18:440-50.

7. Degenhardt L, Ferrari AJ, Calabaria B, Hall WD, Norman RE, McGrath J et al., The global epidemiology and contribution of cannabis use and dependence to the global burden of disease:Results from the GBD 2010 Study. PLoS ONE 8(10): e76635. doi:10.1371/ journal.pone.0076635

8. Erskine HE, Ferrari AJ, Polanczyk GV, Moffitt TE, Murray CJL, Vos $T$ et al., The global burden of conduct disorder and attentiondeficit/hyperactivity disorder in 2010. J Child Psychol Psych. 2014;55:328-36.

9. Ferrari AJ, Charlson FJ, Norman RE, Patten SB, Freedman G, Murray CJL et al., Burden of depressive disorders by country, sex, age, and year: findings from the global burden of disease study 2010 . PLoS Med 10(11): e1001547. doi:10.1371/journal.pmed.1001547

10. Degenhardt L, Baxter AJ, Lee YY, Hall W, Sara GE, Johns N et al., The global epidemiology and burden of psychostimulant dependence: Findings from the Global Burden of Disease Study 2010. Drugs Alcohol Depend. 2014;13:36-47.

11. Jablensky A. Epidemiology of schizophrenia: the global burden of disease and disability. Eur Arch Psychiatry Clin Neurosci. 2000;250;274-85.

12. Whiteford HA, Ferrari AJ, Degenhardt L, Feigin V, Vos T. The global burden of mental, neurological and substance use disorders: An analysis from the global burden of disease. PLoS ONE 10(2): e0116820. doi:10.1371/journal.pone.0116820

13. Charlson FJ, Baxter AJ, Cheng HG, Shidhaye R, Whiteford HA. The burden of mental, neurological, and substance use disorders in China and India: a systematic analysis of community representative epidemiological studies. Lancet 2016;388:376-89.

14. Degenhardt L, Whiteford HA, Ferrari AJ, Baxter AJ, Charlson FJ, Hall WD et al., Global burden of disease attributable to illicit drug use and dependence: findings from the Global Burden of Disease Study 2010. Lancet. 2013;382:1564-74.

15. GBD 2016 Disease and Injury Incidence and Prevalence Collaborators. Global, regional, and national incidence, prevalence, and years lived with disability for 328 diseases and injuries for 195 countries, 1990-2016: a systematic analysis for the Global Burden of Disease Study 2016. Lancet 2017;390:1211-59.
16. Jacob KS. Indian Psychiatry and classification of psychiatric disorders. Indian J Psychiatry 2010;52:S104-9.

17. Baxter AJ, Patton G, Scott KM, Degenhardt L, Whiteford HA. Global epidemiology of mental disorders: What are we missing? PLoS ONE 8(6): e65514. doi:10.1371/journal.pone.0065514

18. Relationship among prevalence, incidence rate, and average duration of disease. Available from: http://sphweb.bumc.bu.edu/otlt/MPHModules/EP/EP713_DiseaseFrequency/EP713_DiseaseFrequency7. html [accessed on 2017 Sep 23]

19. McIntyre RS, Fallu A, Konarski JZ. Measurable outcomes in psychiatric disorders: remission as a marker of wellness. Clin Therap. 2006;28:1882-91.

20. Rubio JM, Olfson M, Villegas L, Peres-Fuentes G, Wang S, Blanco C. Quality of life following remission of mental disorders: Findings from the national epidemiologic survey on alcohol and related condition. J Clin Psychiatry. 2013;74:e449.

21. Haro JM, Novick D, Bertsch J, Karagianis J, Dossenbach M, Jones PB. Cross-national clinical and functional remission rates: Worldwide Schizophrenia Outpatient Health Outcomes (W-SOHO) study. Br J Psychiatry. 2011;199:194-201.

22. Hajda M, Kamaradova D, Latalova K, Prasko J, Ociskova M, Mainerova B et al., Self-stigma, treatment adherence, and medication discontinuation in patients with bipolar disorders in remission - a cross sectional study. Act Nerv Super Rediviva. 2015;57:6-11.

23. Ramasubramanian C, Mohandoss AA, Namasivayam RK. Employability of mentally ill persons in India: A self-report-based population study. Ind Psychiatry J. 2016;25:171-8.

24. Mohandoss AA, Rooban T. An audit of Indian health Insurance claims for mental illness from pooled insurance information bureau's macroindicator data. Indian J Psychol Med. 2017;39:254-61.

25. Lanata A, Valenza G, Nardelli M, Gentili C, Scilingo EP. Complexity index from a personalized wearable monitoring system for assessing remission in mental health. IEEE J Biomed Health Inform. 2015;19:132-9.

26. Mohandoss AA, Thavarajah R. Contribution of Indian psychiatrists to PubMed listed mental health literature during 1995-2013: An exploratory study. Indian J Psychol Med. 2016;38:302-8.

27. Mohandoss AA, Thavarajah R. Contribution and performance of Indian psychiatrists and a Pan-Indian Psychiatry Journal to Mental health literature during 2010-14. J Scientometric Res 2016; 5:100-05. 